\title{
Conversion from temporary to tunneled catheters by nephrologists: report of a single- center experience
}

This article was published in the following Dove Press journal: International Journal of Nephrology and Renovascular Disease II April 2016

Number of times this article has been viewed

\section{Bruno C Silva \\ Camila E Rodrigues \\ Regina CRM Abdulkader \\ Rosilene M Elias}

Nephrology Division, Hospital das Clínicas, University of São Paulo, São Paulo, Brazil
Correspondence: Bruno C Silva Nephrology Division, University of São Paulo, Av. Dr Eneas de Carvalho Aguiar, $255,7^{\circ}$ andar. Cerqueira César, 05403-000, São Paulo, SP, Brazil Tel 55 II 266। 7165

Fax 55 I| 266। 7683

Email brunocaldin@hotmail.com
Background: Nephrologists have increasingly participated in the conversion from temporary catheters (TC) to tunneled-cuffed catheters (TCCs) for hemodialysis.

Objective: To prospectively analyze the outcomes associated with TCC placement by nephrologists with expertise in such procedure, in different time periods at the same center. The impact of vancomycin or cefazolin as prophylactic antibiotics on the infection outcomes was also tested.

Patients and methods: Hemodialysis patients who presented to such procedure were divided into two cohorts: A (from 2004 to 2008) and B (from 2013 to 2015). Time from TC to TCC conversion, prophylactic antibiotics, and reasons for TCC removal were evaluated.

Results: One hundred and thirty patients were included in cohort A and 228 in cohort B. Sex, age, and follow-up time were similar between cohorts. Median time from TC to TCC conversion was longer in cohort A than in cohort B $(14[3 ; 30]$ vs $4[1 ; 8]$ days, respectively; $P<0.0001)$. Infection leading to catheter removal occurred in $26.4 \%$ vs $18.9 \%$ of procedures in cohorts $\mathrm{A}$ and $\mathrm{B}$, respectively, and infection rate was 0.93 vs 0.73 infections per 1,000 catheter-days, respectively $(P=0.092)$. Infection within 30 days from the procedure occurred in $1.4 \%$ of overall cohort. No differences were observed when comparing vancomycin and cefazolin as prophylactic antibiotics on 90-day infection-free TCC survival in a Kaplan-Meier model (log-rank $=0.188$ ). TCC removal for low blood flow occurred in $8.9 \%$ of procedures.

Conclusion: Conversion of TC to TCC by nephrologists had overall infection, catheter patency, and complications similar to data reported in the literature. Vancomycin was not superior to cefazolin as a prophylactic antibiotic.

Keywords: renal dialysis, tunneled catheters, interventional nephrology, infection, temporary catheters, prophylactic antibiotics

\section{Introduction}

Even though native arteriovenous fistulas (AVFs) are considered the best vascular access option for hemodialysis (HD), ${ }^{1}$ tunneled-cuffed catheters (TCCs) are the initial access for $\sim 80 \%$ of patients in the $\mathrm{USA}^{2}$ and $30 \%$ of patients in Europe starting HD. ${ }^{3}$ In fact, for patients with end-stage renal disease (ESRD) admitted with an urgent need for dialysis, the conversion from temporary catheter (TC) to TCC within a brief period might avoid catheter-related infections ${ }^{4}$ and venous thrombosis. ${ }^{5}$ Such conversion, which is usually performed by surgeons and interventional radiologists, can also be done by trained nephrologists ${ }^{6}$ at the centers where interventional nephrology programs have been established. ${ }^{7}$ Bloodstream infection is the main concern when converting TC to TCC and prophylactic antibiotics are routinely used for this reason. However, the routine 
use of narrow-spectrum antibiotics for such procedure might induce bacterial resistance.

In this study, we prospectively evaluated and compared two distinct cohorts of patients who underwent TCC placement without fluoroscopy by different teams of nephrologists, in different periods, at the same center, as part of outcome analysis of our interventional nephrology program. Both teams had the same expertise in TCC placement. As the antibiotic prescription changed from one cohort to another, with reduction of vancomycin and increase of cefazolin prescription, we also compared the impact of the each prophylactic antibiotic on infection outcomes.

\section{Patients and methods}

This study included 358 patients admitted in our service with either dialysis-dependent ESRD or persistent acute kidney injury (defined as at least 3 weeks of dialysis dependence with no prior history of kidney disease), with indication of TCC placement in right internal jugular or femoral vein. Exclusion criteria were: indication for catheter placement in subclavian or left internal jugular veins due to history of thrombosis of right internal jugular vein and anatomic abnormalities that could bring additional risk to the procedure. In these situations, patients were referred to vascular surgeons. Patients were divided into two cohorts according to temporal criteria: cohort A (from 2004 to 2008) and cohort B (from 2013 to 2015). The first cohort corresponds to a prior study, ${ }^{8}$ representing the beginning of TCC placement by nephrologists in our institution, while cohort B corresponds to the recent experience of our interventional nephrology program. In both cohorts, the senior nephrologist had inserted at least 20 TCCs. In addition, the experience and time of training of both senior nephrologists were similar when comparing cohorts A and B. The study was approved by the Ethics Committee for Research Project Analysis - Cappesq (Comissão de Ética para Análise de Projetos de Pesquisa - Cappesq). (CAPPesq \#0033). All patients signed written informed consent.

\section{Techniques of catheter insertion}

All procedures were performed by a trained senior nephrologist and an assistant nephrology trainee in a specific room and under sterile conditions, without fluoroscopy assistance. Superficial sedation with midazolam and fentanyl was provided. TC was converted to TCC by advancing a guidewire through one of the lumens of the existing TC, which was then removed. Afterward, a subcutaneous tunnel was created and the catheter passed through the tunnel using the tunneler provided with the catheter kit. The next step consisted of placing a peel-away sheath/dilator combination over the guidewire.
The dilator and wire were removed and the catheter was inserted centrally through the sheath, which was peeled away. The length of the tunneled catheter inside the vein was based on the chest X-ray performed before conversion, so that the tip of the TCC entered the right atrium. If the insertion site of TC was too high ( $>3 \mathrm{~cm}$ from the clavicle), patients were submitted to de novo TCC placement.

\section{Prophylactic antibiotics}

Prophylactic antibiotics were different in the cohorts. While in cohort $\mathrm{A}$, all patients received intravenous vancomycin (1 g) 1 hour before the procedure, ${ }^{8}$ in cohort B, $58.6 \%$ received the same dose of vancomycin and the remaining patients received intravenous cefazolin (1 g) 1 hour before the procedure. This change in antibiotic prescription was requested by the hospital infectious disease committee 1 year after cohort B initiation, based both on antimicrobial resistance concerns and overall low infection rate of this specific procedure observed in a partial analysis of this cohort.

\section{Follow-up}

Patients were prospectively evaluated after hospital discharge for a minimum period of 90 days and the following outcomes were assessed: death, catheter removal by indication (AVF creation, recovery of native kidney function, conversion to peritoneal dialysis, and kidney transplantation), and removal due to catheter-related reasons (infection or low flux). Catheters still in place were censored at the end of the study period (September 2008 for cohort A and September 2015 for cohort B).

\section{Statistical analysis}

Data are presented as median (25, 75 percentiles). Qualitative variables are expressed as percentages. For continuous variables, we used independent samples $t$-test or the alternative nonparametric Mann-Whitney for comparison between cohorts, or Fisher's exact test for categorical variables. For survival analysis, the Kaplan-Meier model was applied and Cox hazards ratios were calculated. For multivariate analysis, Cox proportional hazard models were used. Statistical significance was defined as a $P$-value $<0.05$. Statistical analyses were performed with Statistical Package for the Social Sciences software version 22 (SPSS Inc., Armonk, NY, USA).

\section{Results}

A total of 358 procedures were performed during the study period. Cohort A comprised 130 patients in a 4-year period (from October 2004 to September 2008), while 228 patients 


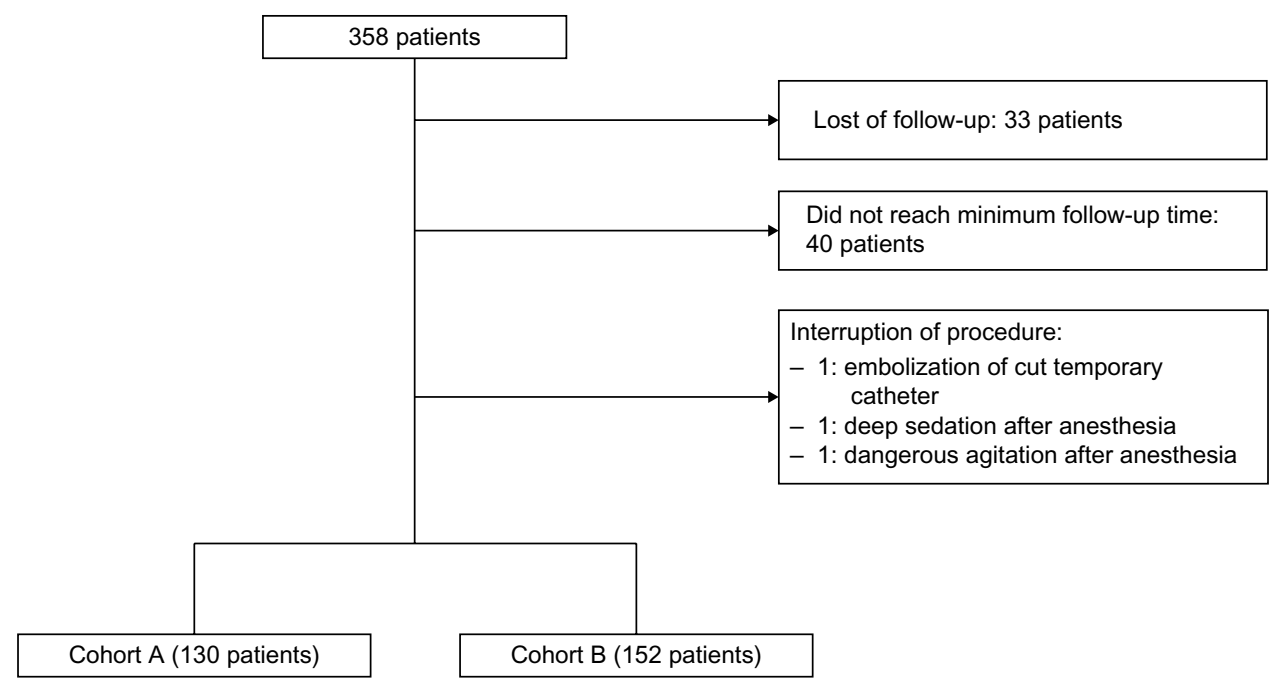

Figure I Cohort description.

were included in cohort B (152 patients included in followup analysis) from October 2013 to September 2015. The inclusion of patients in both cohorts is depicted in Figure 1 and population characteristics are presented in Table 1. In brief, patients were young and comprised mostly men. The etiology of renal disease differed between cohorts, with higher percentage of hypertension, lower percentage of acute kidney injury, and lower percentage of loss of kidney graft

Table I Characteristics of the study population

\begin{tabular}{|c|c|c|c|c|}
\hline Variables & $\begin{array}{l}\text { Whole } \\
\text { cohort } \\
n=358\end{array}$ & $\begin{array}{l}\text { Cohort A } \\
(2004-2008) \\
n=130\end{array}$ & $\begin{array}{l}\text { Cohort B } \\
(20|3-20| 5) \\
n=228\end{array}$ & P-value \\
\hline Included in follow-up analysis & 282 & 130 & 152 & \\
\hline Age (years) & $50.5(35 ; 64)$ & $51.5(38 ; 65)$ & $50(34 ; 63)$ & 0.55 \\
\hline Sex (\% female $)$ & 43.4 & 47.0 & 41.4 & 0.32 \\
\hline Follow-up (days) & $155(75 ; 218)$ & |47 (7I; 264) & $162(76 ; 210)$ & 0.33 \\
\hline Duration of temporary catheter (days) & $5(I ; 12)$ & $14(3 ; 30)$ & $4(1 ; 8)$ & $<0.0001$ \\
\hline Etiology of renal failure (\%) & & & & 0.0001 \\
\hline Glomerulopathy/interstitial disease & 26.3 & 27.7 & 25.4 & \\
\hline Diabetes mellitus & 24 & 29.2 & 21.1 & \\
\hline Hypertension & 17 & 26.2 & 11.8 & \\
\hline Acute kidney injury & 3.4 & 0 & 5.3 & \\
\hline Obstructive/reflux uropathy & 4.7 & 4.6 & 4.8 & \\
\hline Polycystic kidney disease & 3.4 & 1.5 & 4.4 & \\
\hline Loss of kidney graft & 5.3 & 1.5 & 7.5 & \\
\hline Other & 2.2 & 1.5 & 2.6 & \\
\hline Unknown & 13.7 & 7.7 & 17.1 & \\
\hline Catheter brand name (\%) & & & & 0.978 \\
\hline Covidien Permcath ${ }^{\mathrm{TM}}$ & 62.3 & 56.2 & 65.8 & \\
\hline Medcomp Hemoflow ${ }^{\mathrm{TM}}$ & 34.6 & 42.3 & 30.3 & \\
\hline Other & 3.1 & 1.5 & 3.9 & \\
\hline Prophylactic antibiotics (\%) & & & & 0.0001 \\
\hline Cefazolin & 32.6 & 0 & 48.7 & \\
\hline Vancomycin & 67.4 & 100 & 43.8 & \\
\hline Unknown & & 0 & 7.5 & \\
\hline \multicolumn{5}{|l|}{ Catheter length (\%) } \\
\hline $36 \mathrm{~cm}$ & 93.3 & 86.9 & 96.9 & 0.0006 \\
\hline $40 \mathrm{~cm}$ or longer & 6.7 & 13.1 & 3.1 & \\
\hline Vascular site (\%) & & & & 0.084 \\
\hline Internal jugular vein & 97.8 & 95.4 & 99.1 & \\
\hline Femoral vein & $\mathrm{I} .4$ & 2.3 & 0.9 & \\
\hline Other & 0.8 & 2.3 & 0 & \\
\hline
\end{tabular}

Note: Data are presented as median (25, 75 percentiles) or \%, as appropriate. 
in cohorts $\mathrm{A}$ vs $\mathrm{B}(26.2 \%$ vs $11.8 \%, P=0.001 ; 0 \%$ vs $5.3 \%$, $P=0.005$; and $1.5 \%$ vs $7.5 \%, P=0.015$, respectively). Of note, the exchange time from TC to TCC placement was also shorter in cohort B. As expected, the prophylactic antibiotic differed between the cohorts. The rate of TCC insertion increased from 2.7 to 4.8 catheters/month from cohort A to cohort B. At the end of study, 30\% of catheters were still in place in cohort $\mathrm{A}$ and $40.8 \%$ in cohort $\mathrm{B}(P=0.063)$.

\section{Outcomes}

The reason for catheter removal was statistically different when comparing both cohorts $(P=0.0001)$, as shown in Table 2. Cohort B presented higher prevalence of recovery of renal function and higher catheter maintenance for at least 3 months when compared to cohort A. There was no difference between cohorts $\mathrm{A}$ and $\mathrm{B}$ regarding infection-associated catheter removal ( $26.4 \%$ vs $18.9 \%$, respectively, $P=0.092)$ or infection rate $(0.93$ vs 0.73 infections per 1,000 catheters/ day, respectively).

\section{Complications and deaths}

Only one adverse event occurred throughout in both cohorts: an embolization of a cut TC during wire placement. In this case, the procedure was interrupted and the patient immediately referred to the vascular interventionist service of our institution for catheter removal. In two other cases, the procedure was interrupted before starting due to sedationrelated complications (psychomotor agitation in one case and deep sedation in the other case). Overall complication rate was, therefore, was $0.8 \%$.

Overall mortality rate was $17.1 \%$, with a median time of $105(37 ; 232)$ days. The cause of death was not assessed in cohort A. Cardiovascular disease and infection caused $86 \%$ of deaths observed in cohort B. Only one death was attributed to catheter-related infection. Nevertheless, this death occurred 199 days after TCC placement and, thus, was not associated with the procedure.

\section{Catheter infection}

Tunnelitis and catheter-associated bloodstream infection were the events that defined catheter removal due to bacterial infection. Only five catheters were removed within 30 days from TCC insertion, representing $1.4 \%$ of the overall study population. Figure 2 represents the cumulative survival free of infection in the first 90 days from TCC insertion, in a Kaplan-Meier curve, censored by other causes of catheter removal. Despite differences in the conversion time from TC to TCC and in the prophylactic antibiotics, there was no difference in infection rates when cohorts A and B were compared (log-rank $=0.345)$. In a Cox regression analysis, adjusted for prophylactic antibiotics, duration of TC prior to conversion to TCC, age, access site, and diabetes mellitus, there was no factor independently associated with increased catheter infection risk. As the number of events in 90 days limited our analysis, a forward stepwise conditional logistic regression was applied in the beginning to select variables (none of them was significant), followed by an enter method that included antibiotic and cohort (Table 3).

\section{Prophylactic antibiotics}

The cumulative infection-free time after TCC insertion was the outcome used to compare the efficiency of the two distinct antibiotics prescribed. Figure $3 \mathrm{~A}$ represents a Kaplan-Meier curve, which showed no differences when comparing the population that received either vancomycin or cefazolin

Table 2 Outcomes of the study population

\begin{tabular}{llll}
\hline Outcomes & $\begin{array}{l}\text { Whole } \\
\text { cohort } \\
\mathbf{n = 1 8 1}\end{array}$ & $\begin{array}{l}\text { Cohort A } \\
\mathbf{( 2 0 0 4 - 2 0 0 8 )} \\
\mathbf{n = 9 1}\end{array}$ & $\begin{array}{l}\text { Cohort B } \\
\mathbf{( 2 0 1 3 - 2 0 1 5 )} \\
\mathbf{n = 9 0}\end{array}$ \\
\hline $\begin{array}{lll}\mathbf{P} \text {-value } \\
\text { Death (\%) }\end{array}$ & 17.1 & 19.7 & 14.5 \\
$\begin{array}{l}\text { Removal due to catheter-related reason (\%) } \\
\text { Infection }\end{array}$ & 22.6 & 26.4 & 18.9 \\
$\begin{array}{l}\text { Inadequate flux } \\
\text { Removal by indication (\%) }\end{array}$ & 13.8 & 8.9 & 18.9 \\
Arteriovenous fistula creation & & & 30 \\
Method conversion & 32 & 34 & 2.2 \\
Recovery of renal function & 6.1 & 1.1 & 8.9 \\
Kidney transplantation & 5 & 1.1 & 2.2 \\
Other causes & 1.7 & 1.1 & 4.4 \\
\hline
\end{tabular}




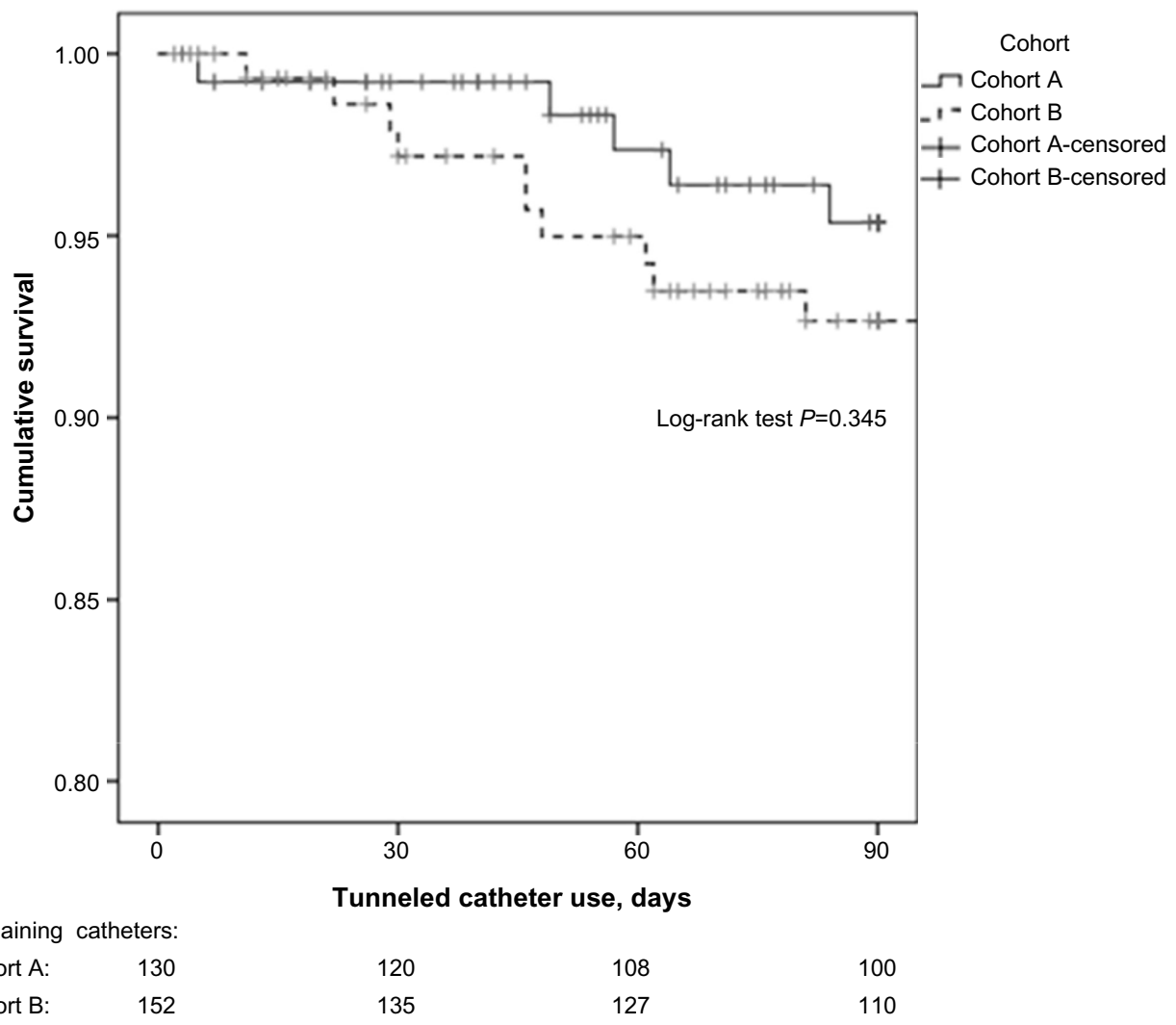

Figure 2 Kaplan-Meier curves for cohorts A and B.

Note: Event: infection leading to catheter removal within 90 days from TCC insertion, censored by other causes of catheter removal.

Abbreviation: TCC, tunneled-cuffed catheter.

(log-rank $=0.188)$. This result was confirmed by a Cox model after adjustment for cohort, diabetes, age, and time spent with the TC before conversion to TCC (Figure 3B), indicating no superior effect of either vancomycin or cefazolin in preventing catheter-related infection.

\section{Discussion}

In this study, we compared the outcomes of TC to TCC conversion at the same center performed by different teams of nephrologists in different periods of time. Even though

Table 3 Cox survival analysis: infection-associated TCC removal

\begin{tabular}{llllll}
\hline $\begin{array}{l}\text { Independent } \\
\text { variable }\end{array}$ & $\mathbf{B}$ & $\mathbf{P}$ & $\operatorname{Exp(B)}$ & $\begin{array}{l}\text { 95\% Cl for } \\
\text { Exp(B) }\end{array}$ \\
\cline { 3 - 6 } & & & & Lower & Upper \\
\hline Cohort A vs cohort B & -1.233 & 0.286 & 0.291 & 0.030 & 2.803 \\
Prophylactic antibiotic & 0.876 & 0.231 & 2.400 & 0.573 & 10.051 \\
\hline
\end{tabular}

Notes: -2 Log likelihood $89.754, P$ of the entire model $=0.22$ I. First step: forward LR with age, diabetes, duration of previous TC before TCC conversion, and access site, and second step with cohort and antibiotics as enter model.

Abbreviations: $\mathrm{Cl}$, confidence interval; TC, temporary catheter; TCC, tunneledcuffed catheter; LR, likelihood ratio. baseline disease, prophylactic antibiotics, and catheter placement rate differed in both the groups, the outcomes were similar, reflecting the reproducibility of the method and making it feasible to be performed by nephrologists. In addition, the choice between vancomycin and cefazolin as the prophylactic antibiotic had no impact on TCC-related infection.

Nephrologists have quite a long story of performing interventions for patients with renal diseases. The AVFs, TCs, and cuffed peritoneal dialysis catheters, for example, were all developed by nephrologists. ${ }^{9-11}$ It has been recognized that nephrologists should provide global care for patients with chronic kidney disease (CKD), not only regarding clinical management, but also by performing procedures such as confection of dialysis access. ${ }^{12}$ Given the actual scenario of increased CKD incidence, this "new nephrologist" could emerge as an option to the well-established expertise of vascular surgeons and interventional radiologists, which in turn avoids fragmented and inadequate care of patients with CKD. Such concern is also present in our institution and, therefore, an intensification of TCC placement has taken place in the past years. The differences found between both 

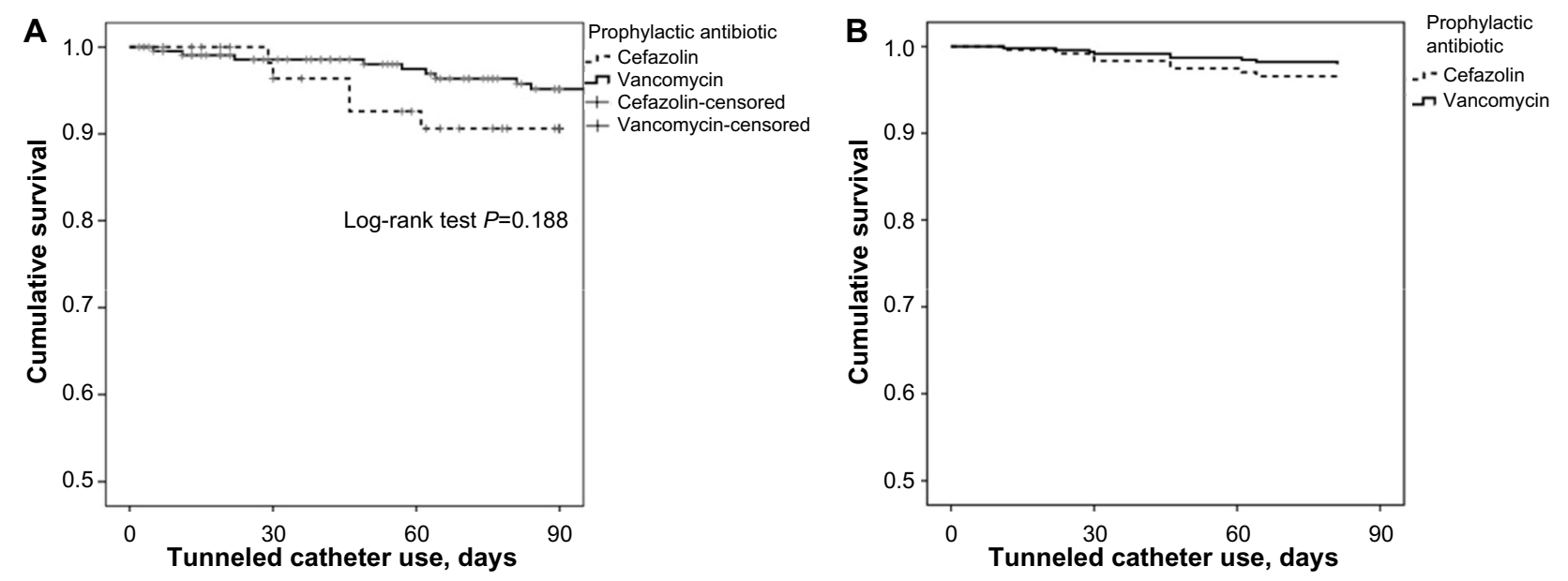

Figure 3 Infection-free TCC survival in the first 90 days according to the prophylactic antibiotics.

Notes: (A) Non-adjusted Kaplan-Meier curves. (B) A Cox regression analysis in a model adjusted for age, diabetes mellitus in cohort A vs B and duration of TC prior to TCC conversion.

Abbreviations: TC, temporary catheter; TCC, tunneled-cuffed catheter.

cohorts reflect the above statement: there was an increase from 2.7 to 4.8 catheters/month from cohort A to cohort B, which reflects the spread of indication to patients with acute kidney injury. Also, the kidney transplantation program of our institution has risen, increasing the number of TCCs for loss of kidney graft as well. It is important to highlight that our center, Hospital das Clinicas, is the largest hospital in Latin America and is characterized by high complex procedures, such as organ transplantations, cardiac, oncologic, and vascular surgeries, and so on. Our population is composed of people not only from nearby cities, but also from many other provinces in Brazil and other nearby countries. As complexity tends to increase along the years, the characteristics of the population covered by our services have changed as well. This might explain why both cohorts were slightly different from each other. Likewise, most of the time, vascular and interventional radiology teams are occupied with complex vascular and endovascular procedures, relegating simple procedures (such as TCC placement) to a lower priority status. ${ }^{7}$ Therefore, interventional nephrology programs could fill such gap in centers with similar characteristics.

The main concern of the conversion from TC to TCC is the risk of infection, which represented $14.5 \%$ of total TCC removal in this study. Nevertheless, only $1.4 \%$ of TCCs were removed within 30 days from insertion. Overall infection rate was as low as 0.8 infections $/ 1,000$ catheter-days. Other studies have reported similar infection rates, ranging from 1.0 to 1.1 infections $/ 1,000$ catheter-days. ${ }^{13-15}$ Falk et a ${ }^{16}$ have reported even higher 30-day infection incidence (9.4\%) and rate (3/1,000 catheter-days). Recently, Bajaj et al ${ }^{17}$ have reported overall infection incidence of $15.7 \%$ and an infection rate of 1.9/1,000 catheter-days. Therefore, overall infection risk due to this specific procedure reported by this study and other studies ${ }^{8,15-17}$ is similar to that of de novo catheter placement.

The efficacy of prophylactic antibiotic administration to prevent infections in TCC procedures is still controversial. A meta-analysis that evaluated oncologic patients found no superiority of using prophylactic antibiotics (vancomycin, teicoplanin, or ceftazidime) over no use of such medications. ${ }^{18}$ In a study conducted in ESRD patients who underwent TCC placement, cefazolin significantly reduced catheter-related infections, bacteremia, and catheter loss over placebo. ${ }^{19} \mathrm{In}$ our center, we routinely use prophylactic antibiotics for such procedure, according to an institutional protocol developed previously and reported elsewhere. ${ }^{8}$ For the first cohort and initially for the second cohort, only vancomycin was used. Afterward, the institutional infectious disease committee requested the prescription of cefazolin for such procedure. We found no differences regarding infection outcomes when comparing both antibiotics, even after adjustment for cohort, diabetes, age, and time spent with TC before conversion to TCC. This result highlights an obvious advantage of cefazolin over vancomycin in avoiding induction of bacterial resistance. As far as we are concerned, this is the first study to make such a comparison.

Catheter patency is another important outcome when evaluating HD catheters. Specifically regarding TCCs, there are two major aspects that might influence blood flow: catheter kinking in transition of the tunneled portion to the 
venous entry site and the catheter tip position, which should be in the right atrium in order to optimize blood flow. ${ }^{20}$ In this study, only $8.9 \%$ of TCCs were removed for inadequate flux. Other studies have reported patency rates varying from $42 \%$ to $83 \%{ }^{14,16,21,22}$ This suggests that relying on previous thorax X-ray to estimate the catheter tip position might be an interesting option when fluoroscopy is not available. Also, not placing TCC when venotomy is too high $(>3 \mathrm{~cm}$ from the clavicle, in our protocol) is another important measure for avoiding catheter kinking and, therefore, promoting better catheter patency.

Conversion from TC to TCC is a safe procedure when performed by nephrologists at a hospital environment. In this study, only three procedure-associated complications were reported $(0.8 \%$ of all procedures). Only one of them required assistance of the interventional radiology team of our institution. Other studies have also reported low complication index..$^{8,13,15,16,21}$ Even though we did not use fluoroscopy in our procedures for limitations of our service, the importance of having an interventional radiology or vascular backup team must be highlighted in order to deal with the complications that cannot be managed by nephrologists.

Finally, the mortality rates in cohorts A and B, respectively, were $19.7 \%$ and $14.5 \%(P=0.183)$, observed in a median period of $147(71 ; 264)$ vs $162(76 ; 210)$ days. Even though the annual mortality rate of Brazilian patients on HD has been stable in the past years (at $\sim 18 \%$ ), ${ }^{23}$ we do not have national data regarding mortality during the first year of HD. As a comparison, in the USA, survival during the first year after HD initiation with a catheter was $78 \%$, which was lower than that of patients who started with an AVF (89\%). ${ }^{2}$ An important characteristic of our population that must be pointed out is that virtually all patients initiated HD in an emergency environment, which might explain such relatively high mortality rate.

\section{Conclusion}

This study represents the experience of the nephrology service of our institution regarding the conversion from TC to TCC. Overall infection, catheter patency, and complications are similar to data reported in the literature. Also, vancomycin or cefazolin used as prophylactic antibiotics had similar impact on catheter infection rate. The safety of such procedure and the relatively high risks of venous thrombosis and infection associated with TC reinforce the importance of incorporating such a technique in the routine practice of a nephrologist, as part of the optimal care of patients with CKD.

\section{Acknowledgment}

The authors acknowledge Alcino Pires Gama and Simone Chinwa Lo for their valuable contribution to this study.

\section{Disclosure}

The authors report no conflicts of interest in this work.

\section{References}

1. Lacson E Jr, Lazarus JM, Himmelfarb J, Ikizler TA, Hakim RM. Balancing fistula first with catheters last. Am J Kidney Dis. 2007:50; 379-395.

2. Malas MB, Canner JK, Hicks CW, et al. Trends in incident hemodialysis access and mortality. JAMA Surg. 2015;150:441-448.

3. Pisoni RL, Young EW, Dykstra DM, et al. Vascular access use in Europe and the United States: results from the DOPPS. Kidney Int 2002;61:305-316.

4. Mermel LA. Prevention of intravascular catheter-related infections. Ann Intern Med. 2000;132:391-402.

5. MacRae JM, Ahmed A, Johnson N, Levin A, Kiaii M. Central vein stenosis: a common problem in patients on hemodialysis. ASAIO $J$. 2005;51:77-81.

6. Sachdeva B, Abreo K. The history of interventional nephrology. Adv Chronic Kidney Dis. 2009;16:302-308.

7. The New Nephrologist. Am J kidney Dis. 2000;35:978-979.

8. Motta Elias R, da Silva Makida SC, Abensur H, et al. Insertion of tunneled hemodialysis catheters without fluoroscopy. $J$ Vasc Access. 2010;11:138-142.

9. Tenckhoff H, Schechter H. A bacteriologically safe peritoneal access device. Trans Am Soc Artif Intern Organs. 1968;14:181-187.

10. Brescia MJ, Cimino JE, Appel K, Hurwich BJ. Chronic hemodialysis using venipuncture and a surgically created arteriovenous fistula. $N$ Engl J Med. 1966;275:1089-1092.

11. Uldall PR, Woods F, Bird M, Dyck R. Subclavian cannula for temporary hemodialysis. Proc Clin Dial Transplant Forum. 1979;9:268-272.

12. Asif A, Byers P, Vieira F, Roth D. Developing a comprehensive diagnostic and interventional nephrology program at an academic center. Am J Kidney Dis. 2003;42:229-233.

13. Duszak R Jr, Haskal ZJ, Thomas-Hawkins C, et al. Replacement of failing tunneled hemodialysis catheters through pre-existing subcutaneous tunnels: a comparison of catheter function and infection rates for de novo placements and over-the-wire exchanges. JVasc Interv Radiol. 1998;9:321-327.

14. Garofalo RS, Zaleski GX, Lorenz JM, Funaki B, Rosenblum JD, Leef JA. Exchange of poorly functioning tunneled permanent hemodialysis catheters. AJR Am J Roentgenol. 1999;173:155-158.

15. Van Ha TG, Fimmen D, Han L, Funaki BS, Santeler S, Lorenz J. Conversion of non-tunneled to tunneled hemodialysis catheters. Cardiovasc Intervent Radiol. 2007;30:222-225.

16. Falk A, Prabhuram N, Parthasarathy S. Conversion of temporary hemodialysis catheters to permanent hemodialysis catheters: a retrospective study of catheter exchange versus classic de novo placement. Semin Dial. 2005;18:425-430.

17. Bajaj SK, Ciacci J, Kirsch M, Ebersole JD. A single institutional experience of conversion of non-tunneled to tunneled hemodialysis catheters: a comparison to de novo placement. Int Urol Nephrol. 2013;45: $1753-1759$.

18. van de Wetering MD, van Woensel JB, Lawrie TA. Prophylactic antibiotics for preventing Gram positive infections associated with longterm central venous catheters in oncology patients. Cochrane Database Syst Rev. 2013;11:CD003295.

19. Huddam B, Azak A, Kocak G, Ortabozkoyun L, Duranay M. The efficacy of prophylactic antibiotics administration prior to insertion of tunneled catheter in hemodialysis patients. Ren Fail. 2012;34:998-1001. 
20. Vesely TM. Central venous catheter tip position: a continuing controversy. J Vasc Interv Radiol. 2003;14:527-534.

21. Obialo CI, Conner AC, Lebon LF. Tunneled hemodialysis catheter survival: comparison of radiologic and surgical implantation. ASAIOJ. 2000;46:771-774.

22. Shingarev R, Barker-Finkel J, Allon M. Natural history of tunneled dialysis catheters placed for hemodialysis initiation. J Vasc Int Radiol. 2013;24:1289-1294.
23. Sesso RC, Lopes AA, Thome FS, Lugon JR, Dos Santos DR. Brazilian Chronic Dialysis Survey 2013 - trend analysis between 2011 and 2013. J Bras Nefrol. 2014;36:476-481.

\section{Publish your work in this journal}

The International Journal of Nephrology and Renovascular Disease is an international, peer-reviewed open-access journal focusing on the pathophysiology of the kidney and vascular supply. Epidemiology, screening, diagnosis, and treatment interventions are covered as well as basic science, biochemical and immunological studies. The journal welcomes original research, clinical studies, reviews \& evaluations, expert opinion and commentary, case reports and extended reports. The manuscript management system is completely online and includes a very quick and fair peerreview system, which is all easy to use. Visit http://www.dovepress.com/ testimonials.php to read real quotes from published authors.

Submit your manuscript here: http://www.dovepress.com/international-journal-of-nephrology-and-renovascular-disease-journal 